\title{
Zoledronic acid reverses cisplatin resistance in nasopharyngeal carcinoma cells by activating the mitochondrial apoptotic pathway
}

\author{
YANJIE YOU ${ }^{*}$, HAIJUN LI $^{2 *}$, JIONGYU CHEN $^{3}$, XIN QIN $^{4}$ and YONGGANG RAN ${ }^{5}$ \\ ${ }^{1}$ Pathological Examination and Research Center, Luohe Medical College, Luohe, Henan 462002; \\ ${ }^{2}$ Department of Radiation Oncology, The Second People's Hospital of Neijiang, Neijiang, Sichuan 641000; \\ ${ }^{3}$ Department of Laboratory, Cancer Hospital of Shantou University, Shantou, Guangdong 515041; \\ ${ }^{4}$ Medical College, Hubei University of Arts and Science, Xiangyang, Hubei 441053; \\ ${ }^{5}$ Department of Teaching and Training, Bethune Military Medical NCO Academy of PLA, \\ Shijiazhuang, Hebei 050081, P.R. China
}

Received April 30, 2015; Accepted September 9, 2016

DOI: $10.3892 / 01.2017 .5611$

\begin{abstract}
Despite improvements to radiotherapeutic strategies, resistance to adjuvant chemotherapy remains the main problem underlying the low 5-year survival rate in patients with nasopharyngeal carcinoma (NPC). In the present study, the human NPC cell line HNE1 was exposed to gradually increasing concentrations of cisplatin (CDDP) in order to establish a drug-resistant sub-cell line, HNE1/CDDP. HNE1/CDDP cells exhibited multidrug resistance and a prolonged doubling time, as compared with the parent HNE1 cells. Furthermore, pretreatment with zoledronic acid (ZOL) appeared to resensitize the CDDP-resistant cells by inducing $\mathrm{S}$-phase cell cycle arrest and the mitochondrial apoptotic pathway by upregulating the expression of B-cell lymphoma-2 (BCL-2)-associated $\mathrm{X}$ protein and caspase- 9 and downregulating the expression of BCL-2. The results of the present study suggested that HNE1/CDDP cells are a stable, multidrug-resistant NPC cell line that may serve as an important tool for research in drug resistance. In addition, the application of ZOL may hold clinical therapeutic potential for the treatment of drug resistance in NPC.
\end{abstract}

\section{Introduction}

Nasopharyngeal carcinoma (NPC) is a subtype of head and neck cancers with a remarkable racial and geographical distribution (1-3). The annual incidence of NPC is 25-50 cases

Correspondence to: Dr Yanjie You, Pathological Examination and Research Center, Luohe Medical College, 148 Daxue-Road, Luohe, Henan 462002, P.R. China

E-mail: youyanjie@163.com

*Contributed equally

Key words: nasopharyngeal carcinoma, chemotherapy, drug resistance, cisplatin, zoledronic acid per 100,000 people in Southern China, which is 100-fold higher than in western countries (3). Currently, radiotherapy is the most common therapeutic strategy for NPC, although chemotherapy is used as an adjuvant to treat advanced carcinoma and to enhance the radiosensitivity $(4,5)$. However, the success of chemotherapy is dependent on the sensitivity of the tumor to antineoplastic agents (5). To prevent drug resistance and recurrence, chemotherapeutic regimes must effectively induce the apoptosis of cancer cells. Although some patients initially respond to chemotherapy, the majority of patients with advanced tumors experience treatment failure, as NPC cells often acquire resistance to drugs and may develop multidrug resistance (4-8).

To date, studies on drug resistance have been limited to only a few types of cancers (9-11). It is unclear whether the drug resistance mechanisms in NPC cells are common to other types of cancer or are unique. Therefore, the establishment of a drug-resistant NPC cell line in vitro, by exposure to the antitumor agent, is important to elucidate the biological mechanisms underlying drug resistance and to develop reliable methods for reversing drug resistance.

Cisplatin (CDDP), which is a chemotherapeutic agent that is able to induce dsDNA breaks, is one of the most active drugs for the treatment of NPC (4-8). Notably, radiotherapy and adjuvant CDDP chemotherapy have emerged as the standard therapeutic strategy for NPC $(11,12)$. However, in previous studies, CDDP-based chemotherapy frequently resulted in acquired resistance in NPC cancer cells $(7,8,11-14)$. The resistance to chemotherapeutic agents has been associated with a failure to induce apoptosis $(7,13,14)$. To the best of our knowledge, few studies have investigated CDDP-resistant NPC cell lines $(15,16)$.

The present study established a CDDP-resistant NPC cell line by continuously increasing the concentration of CDDP, and evaluated the biological characteristics of this drug-resistant cell line. Furthermore, it was demonstrated that zoledronic acid (ZOL), a third-generation bisphosphonate, was able to reverse CDDP resistance in NPC cells by inducing S-phase cell cycle arrest and subsequently reactivating the mitochondrial apoptotic pathway. 


\section{Materials and methods}

Reagents. CDDP and vinorelbine were purchased from Jiangsu Hansoh Pharmaceutical Co., Ltd. (Lianyungang, China) and stored at a concentration of $1 \mathrm{mg} / \mathrm{ml}$ at room temperature (RT) and a concentration of $10 \mathrm{mg} / \mathrm{ml}$ at $4^{\circ} \mathrm{C}$, respectively. Carboplatin was obtained from Bristol-Myers Squibb (New York, NY, USA) and stored at a concentration of $10 \mathrm{mg} / \mathrm{ml}$ at RT. 5-fluorouracil was purchased from Shanghai Xudong Haipu Pharmaceuticals Ltd. (Shanghai, China) and stored at a concentration of $25 \mathrm{mg} / \mathrm{ml}$ at RT. Docetaxel was purchased from Wanma Pharmaceutical, Co., Ltd. (Hangzhou, China) and stored at a concentration of $40 \mathrm{mg} / \mathrm{ml}$ at $4^{\circ} \mathrm{C}$. Arsenic trioxide was purchased from Yierda Pharmaceuticals, Co., Ltd. (Harbin, China) and stored at the concentration of $1 \mathrm{mg} / \mathrm{ml}$ at $4^{\circ} \mathrm{C}$. Oxaliplatin, etoposide, cyclophosphamide and irinotecan were purchased from Jiangsu Hengrui Medicine, Co., Ltd. (Lianyungang, China), and nedaplatin was obtained from Qilu Pharmaceutical, Co., Ltd. (Jinan, China); these agents were stored at pre-designed concentrations diluted in $0.9 \% \mathrm{NaCl}$ at $\mathrm{RT}$. ZOL, which was provided by Novartis Pharma AG (Basel, Switzerland), was dissolved in PBS and maintained at $-20^{\circ} \mathrm{C}(8)$. All dose formulations were prepared on the day of usage.

Cell lines and culture conditions. The HNE1/CDDP cell line (The University of Hong Kong, Pokfulam, Hong Kong), was generated by continuously exposing parental HNE1 cells to gradually increasing concentrations of CDDP. The initial concentration of CDDP was $0.02 \mu \mathrm{g} / \mathrm{ml}$ and the final concentration was $1 \mu \mathrm{g} / \mathrm{ml}$. HNE1 and HNE1/CDDP cells were cultured in Dulbecco's modified Eagle's medium (DMEM) supplemented with 10\% fetal bovine serum (both Gibco; Thermo Fisher Scientific, Inc., Waltham, MA, USA) in a humidified incubator containing 5\% $\mathrm{CO}_{2}$ at $37^{\circ} \mathrm{C}$. The cells were cultured under these conditions for 1 month prior to performing the experiments.

Drug sensitivity assay. The sensitivity of the HNE1 and HNE1/CDDP cells to CDDP was assessed using MTT colorimetric assays, as described previously (17). Briefly, the cells were plated in triplicate onto 96 -well plates at a density of $4 \times 10^{3}$ cells/well and, after 1 day, the cells were exposed to CDDP at increasing concentrations. MTT assays were performed after $48 \mathrm{~h}$ of incubation. The cells were then treated with ZOL $(0,0.5,1,2,4$ and $8 \mu \mathrm{g} / \mathrm{ml})$ and cell growth was measured after $48 \mathrm{~h}$. Controls were treated with a dimethyl sulfoxide (DMSO) at concentrations equal to that in drug-treated cells. After $48 \mathrm{~h}$, cells were treated with $20 \mu \mathrm{l}$ MTT (Sigma-Aldrich; Merck Millipore, Darmstadt, Germany) at $37^{\circ} \mathrm{C}$ for $4 \mathrm{~h}$. The supernatants were then removed and the formazan crystals in each well were solubilized by the addition of $150 \mu \mathrm{l}$ DMSO. The formazan product was measured at a wavelength of $490 \mathrm{~nm}$ using a microplate reader. The half maximal inhibitory concentration $\left(\mathrm{IC}_{50}\right)$ was identified as the concentration of drug required to achieve 50\% growth inhibition relative to control populations. The resistance index (RI) was defined as the $\mathrm{IC}_{50}$ of HNE1/CDDP cells divided by that of parental HNE1 cells.

Analysis of morphological changes. Exponentially growing HNE1 and HNE1/CDDP cells were transferred to 6-well plates and allowed to reattach for at least $24 \mathrm{~h}$. Images of the cells captured under a light microscope (Olympus IX70; Olympus Corporation, Tokyo, Japan).

Growth curve analysis. Single-cell suspensions were prepared from the cells in the exponential growth phase. Aliquots containing $4 \times 10^{3}$ cells/well were seeded in triplicate into 12 -well plates containing $1 \mathrm{ml}$ DMEM. Cell counts were conducted every $24 \mathrm{~h}$ for 5 days. The doubling time (Td) of each cell line was counted according to the formula $\mathrm{Td}=\mathrm{T} \times \lg 2 /(\lg \mathrm{N} 2-\operatorname{lgN} 1)$, where $\mathrm{N} 1$ is the cell number at the beginning and $\mathrm{N} 2$ is the cell number at the end, usually the end of the exponential phase.

Cell cycle and apoptosis analyses by flow cytometry. Cells were harvested and fixed in $70 \%$ ethanol overnight at $-20^{\circ} \mathrm{C}$, after which they were suspended in $500 \mu \mathrm{l}$ staining solution [50 $\mu \mathrm{g} / \mathrm{ml}$ propidium iodide (PI), $100 \mu \mathrm{g} / \mathrm{ml}$ RNAase and $0.2 \%$ Triton X-100] for $30 \mathrm{~min}$. The DNA content and percentage of cells at each phase of the cell cycle were calculated using the Cytomics FC 500 flow cytometer (Beckman Coulter, Inc., Brea, CA, USA). For the analysis of apoptosis, the cells were stained with Annexin V/PI using an Annexin V-Fluorescein Isothiocyanate Apoptosis Detection kit (Tiangen Biotech, Co., Ltd., Beijing, China) and then subjected to flow cytometry, according to the manufacturer's protocol.

Western blot analysis. The cells were lysed using RIPA lysis buffer (Tiangen Biotech Co., Ltd.) and 50 $\mu \mathrm{g}$ of total protein from each sample was separated by $12 \%$ SDS-PAGE, followed by transfer onto a polyvinylidene difluoride membrane. The membrane was blocked overnight at $4^{\circ} \mathrm{C}$ using $5 \%$ bovine serum albumin (Sigma-Aldrich; Merck Millipore) in PBS. Subsequently, the membrane was incubated with the following primary antibodies overnight at $4^{\circ} \mathrm{C}$ : Anti-ATP-binding cassette (ABC) subfamily B member 1 (ABCB1)/P-glycoprotein (cat. no. ab129450; 1:400; Abcam, Cambridge, UK), anti-ABC subfamily C member $1(\mathrm{ABCC} 1) / \mathrm{multidrug}$ resistance-associated protein 1 (MRP1) (cat. no. ab32574; 1:400; Abcam), anti-ABC subfamily $G$ member 2 (ABCG2)/breast cancer resistance protein (BCRP) (ab63907; 1:200; Abcam), anti-B-cell lymphoma-2 (BCL-2)-associated X protein (BAX) (cat. no. sc-7480; 1:200; Santa Cruz Biotechnology, Inc., Santa Cruz, CA, USA) and anti-BCL-2 (cat. no. sc-492; 1:200; Santa Cruz Biotechnology, Inc.). After washing with $0.1 \%$ Tween-20 in PBS, the membranes were then incubated with horseradish peroxidase-conjugated goat anti-rabbit IgG secondary antibody (cat. no. sc-2004; 1:2,000; Santa Cruz Biotechnology, Inc.) for $1 \mathrm{~h}$ at $37^{\circ} \mathrm{C}$. Proteins were detected using an enhanced chemiluminescence kit (GE Healthcare Life Sciences, Chalfont, UK). The blots were reprobed with a GAPDH monoclonal antibody (cat. no. ab9485; 1:2,500; Abcam) to confirm equal loading of the different samples.

Statistical analysis. Data are presented as the mean \pm standard deviation of at least three repeated experiments. Data were analyzed using Student's t-test in the SPSS 16.0 software package (SPSS, Inc., Chicago, IL, USA). P $<0.05$ was considered to indicate a statistically significant difference. 
Table I. Multidrug sensitivity of HNE1 and HNE1/CDDP cells.

\begin{tabular}{lcrr}
\hline & \multicolumn{3}{c}{$\mathrm{IC}_{50}$ values $(\mu \mathrm{g} / \mathrm{ml})$} \\
\cline { 2 - 4 } Drug & HNE1 & HNE1/CDDP & \\
\hline CDDP & $1.20 \pm 0.03$ & $7.00 \pm 0.19$ & 5.83 \\
Vinorelbine & $28.13 \pm 0.78$ & $28.13 \pm 0.82$ & 1.00 \\
Carboplatin & $17.50 \pm 0.53$ & $46.70 \pm 1.78$ & 2.67 \\
5-Fluorouracil & $0.27 \pm 0.01$ & $0.58 \pm 0.03$ & 2.15 \\
Oxaliplatin & $0.83 \pm 0.05$ & $3.26 \pm 0.09$ & 3.93 \\
Etoposide & $3.30 \pm 0.06$ & $14.00 \pm 0.38$ & 4.24 \\
Cyclophosphamide & $5828.82 \pm 104.36$ & $13207.91 \pm 387.04$ & 2.27 \\
Irinotecan & $21.50 \pm 0.63$ & $208.60 \pm 7.19$ & 9.70 \\
Nedaplatin & $8.11 \pm 0.31$ & $9.34 \pm 0.44$ & 2.56 \\
Docetaxel & $11.14 \pm 0.47$ & $35.30 \pm 0.89$ & 0.84 \\
Arsenic trioxide & $7.32 \pm 0.28$ & $20.07 \pm 0.52$ & 4.82 \\
Zoledronic acid & $18.56 \pm 0.40$ & & \\
\hline
\end{tabular}

CDDP, cisplatin; $\mathrm{IC}_{50}$, half maximal inhibitory concentration; $\mathrm{RI}$, resistance index.
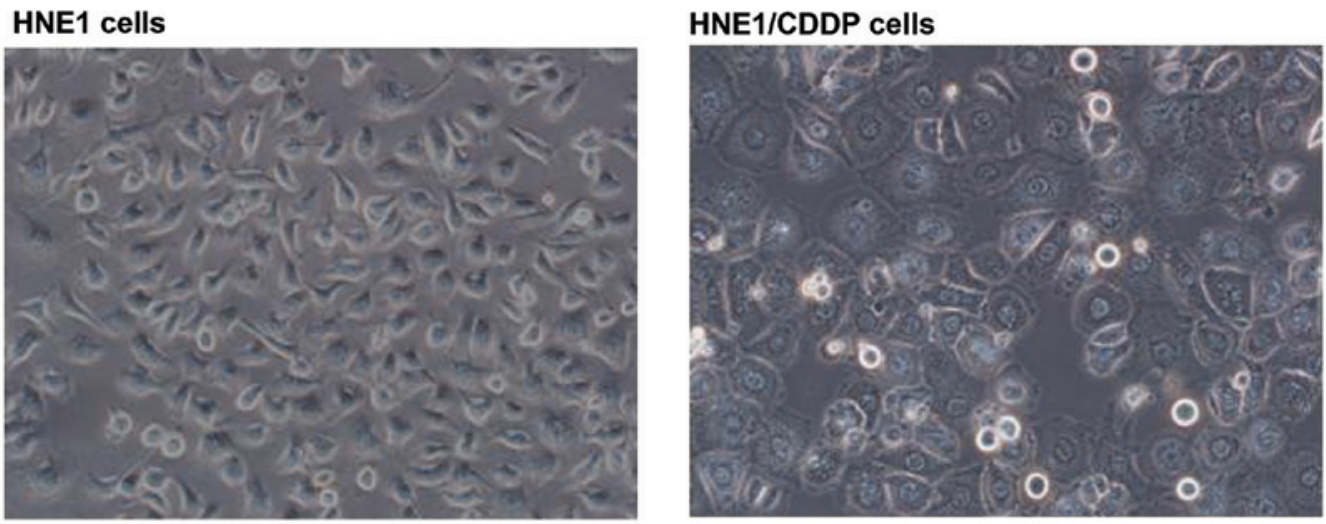

Figure 1. Morphology of HNE1 and HNE1/CDDP cells in the exponential growth phase. Morphological characteristics were determined using an inverted microscope at an original magnification of x200. HNE1 cells were relatively uniform in size and polygonal-shaped, while HNE1/CDDP cells showed an irregular shape and size, with both giant and small cells apparent. CDDP, cisplatin.

\section{Results}

Establishment and phenotype of HNE1/CDDP cells. The establishment of the CDDP-resistant sub cell line, HNE1/CDDP, was achieved over a period of 10 months by increasing the concentration of CDDP that the HNE1 parental cells were exposed to. HNE1/CDDP cells were then cultured in drug-free medium for subsequent analyses. The stability of the resistance to CDDP was determined by examining the $\mathrm{IC}_{50}$ at monthly intervals during the period of culturing in drug-free medium. The drug sensitivity profiles of the HNE1 and HNE1/CDDP cells are shown in Table I. Notably, the HNE1/CDDP cells exhibited cross-resistance to several other drugs to which they were not exposed. This observation suggests that HNE1/CDDP cells are a multidrug-resistant cell line. However, the chemosensitivity of HNE1/CDDP cells was not significantly affected when they were treated with vinorelbine, docetaxel or ZOL, as compared with the parental HNE1 cells.
Morphological changes of HNE1/CDDP cells and cell growth curves. HNE1 and HNE1/CDDP cells were adherent, epithelial-like and polygon-shaped. HNE1 cells had a relatively uniform cell size and shape. Conversely, HNE1/CDDP cells had an irregular shape and varied in size; both giant and small cells were observed (Fig. 1). HNE1 cells were in the logarithmic growth phase on the day 1 after seeding, while HNE1/CDDP cells exhibited a markedly slower growth curve compared with the parental HNE1 cells, probably due to lower cell adherence (Fig. 2). According to the cell growth curves, the Tds of HNE1 and HNE1/CDDP cells were $37.44 \pm 1.34$ and $41.85 \pm 0.89 \mathrm{~h}$, respectively, which was significantly different $(\mathrm{P}<0.05)$.

$Z O L$ pretreatment partially reverses the $C D D P$ resistant phenotype. Previous studies reported that low doses of ZOL exhibited synergistic functions in combination with other chemotherapeutic agents in various cancer cell lines (17-19). 
Therefore, in the present study, HNE1 cells were treated with $2.90 \mu \mathrm{g} / \mathrm{ml} \mathrm{ZOL}(10 \mu \mathrm{M})$ for $6 \mathrm{~h}$ prior to CDDP exposure. First, whether pretreatment with ZOL was able to reverse the CDDP resistance in NPC cells was assessed. Both HNE1 and HNE1/CDDP cells were pretreated with ZOL for $6 \mathrm{~h}$, and then exposed to various doses of CDDP for another $48 \mathrm{~h}$. As shown in Fig. 3, pretreatment with ZOL alone resulted in a small inhibitory effect on the cell growth of HNE1 and HNE1/CDDP cells, suggesting its potential use as a sensitizer for chemotherapy. The CDDP-mediated growth inhibition of HNE1 cells was not significantly increased by pretreatment with ZOL. However, pretreatment with ZOL significantly augmented the inhibitory effect of CDDP on HNE1/CDDP cells $(\mathrm{P}<0.05$; Fig. 3$)$. The $\mathrm{IC}_{50}$ of CDDP in HNE1/CDDP cells decreased from $7.00 \pm 0.19$ to $3.84 \pm 0.13 \mu \mathrm{g} / \mathrm{ml}$ and the RI decreased from 5.83 to 3.2 ( $\mathrm{P}<0.01$ for both).

ZOL pretreatment increases $S$-phase arrest and cell apoptosis. The cell cycle distribution and DNA content of HNE1 and HNE1/CDDP cells was assessed by flow cytometry. As demonstrated in Fig. 4, a prominent arrest at the G0/G1 phase occurred in HNE1/CDDP cells, as compared with HNE1 cells $(45.34 \pm 3.33$ vs. $25.72 \pm 2.87 \%$, respectively; $\mathrm{P}<0.05)$. Correspondingly, there was a significant decrease in the percentage of HNE1/CDDP cells in the $S$ phase, as compared with HNE1 cells (41.20 \pm 6.12 vs. $56.74 \pm 6.17 \%$, respectively; $\mathrm{P}<0.01)$. Exposure to $1 \mu \mathrm{g} / \mathrm{ml}$ CDDP induced an increase in the percentage of HNE1 cells, but not HNE1/CDDP cells, in the G2/M phase. Notably, pretreatment with $\mathrm{ZOL}$ resulted in a decrease in the percentage of HNE1/CDDP cells in the G0/G1 phase (down to $21.05 \pm 3.73 \%$ ), followed by an increase in the percentage of cells in the $\mathrm{S}$ phase (up to $57.27 \pm 6.33 \%$ ), as compared with CDDP treatment alone $(\mathrm{P}<0.05)$. However, this alteration following combined treatment with CDDP and ZOL was not observed in the HNE1 cells.

It is generally accepted that CDDP reduces the viability of cancer cells by inducing apoptosis (11-14). Therefore, the present study further examined whether ZOL pretreatment promoted apoptosis in CDDP-resistant cells. As shown in Fig. 5, combined treatment with ZOL and CDDP did not significantly increase the percentage of apoptotic HNE1 cells, as compared with CDDP treatment alone $(27.8 \pm 3.78$ vs. $25.7 \pm 2.93 \%$, respectively). However, combined treatment with ZOL and CDDP significantly increased the proportion of apoptotic HNE1/CDDP cells, as compared with HNE1/CDDP cells treated with CDDP alone $(21.5 \pm 2.73$ vs. $7.3 \pm 1.74 \%$, respectively; $\mathrm{P}<0.05$; Fig. 5). These results suggest that reversal of CDDP resistance by ZOL may be due to rescued $\mathrm{S}$ phase arrest and subsequent promotion of apoptosis.

Expression pattern of drug resistance and apoptosis-related genes. The expression of several important genes associated with drug resistant was determined by western blotting. Since the mitochondrial apoptotic pathway has previously been associated with the sensitivity of cancer cells to $\operatorname{CDDP}(20,21)$, the expression statuses of BCL-2, BAX and caspase-9 were also evaluated in the present study. As shown in Fig. 6, pretreatment with ZOL did not affect the expression of drug resistant-related genes in the HNE1 and HNE1/CDDP cells. However, exposure of HNE1/CDDP cells to ZOL plus CDDP resulted in upregulated expression of BAX and caspase-9,

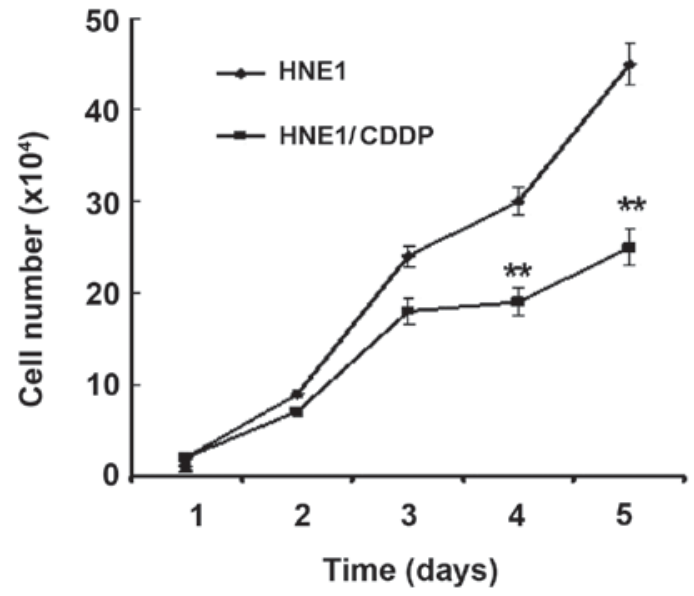

Figure 2. Growth curves of HNE1 and HNE1/CDDP cells. Cells were cultured in 12 -well plates at a density of $4 \times 10^{3}$ cells per well in triplicate. Cell counts were performed every $24 \mathrm{~h}$ for 5 days. Data are expressed as the mean \pm standard deviation of three independent experiments. ${ }^{* *} \mathrm{P}<0.01$ vs. the HNE1 cells. CDDP, cisplatin.

and downregulated expression of BCL-2, as compared with CDDP treatment alone. Conversely, modulation of the apoptosis-related genes by ZOL was minimal in HNE1 cells.

\section{Discussion}

The success of chemotherapy depends on the sensitivity of the tumor to anticancer agents. The establishment of drug-resistant cancer cell lines is crucial for elucidating the biological characteristics and drug resistance mechanisms of resistant cancer cells $(7,9,10,12)$. The present study successfully established a CDDP-resistant NPC cell line by gradually increasing the concentration of CDDP in the cell culture medium over a period of 10 months. After 3 months of culturing in drug-free medium, the resistance of the HNE1/CDDP cells to CDDP was shown to be stable, indicating that these cells were an appropriate model for NPC drug resistance. Notably, the HNE1/CDDP cells had a high resistance to other platinum-based drugs, including carboplatin, oxaliplatin and nedaplatin, as compared with the parental cells. In addition, the CDDP-resistant cell line exhibited cross-resistance to other structurally and mechanistically different drugs, including etoposide, irinotecan, cyclophosphamide and arsenic trioxide. This characteristic of drug-induced activation of multidrug resistance suggests that chemotherapy combinations of CDDP with other chemotherapeutic agents may fail in clinical practice.

Morphological analyses revealed that the HNE1/CDDP cells showed some differences from the parental HNE1 cells in terms of cell shape, size and cytoplasmic vacuoles. Morphological alterations that occur in drug-resistant cells may reflect the survival mechanisms adopted by the cells, including directly increasing energy production/metabolism and DNA repair, or indirectly modulating the signal transduction pathways leading to cell death $(22,23)$. These changes may further lead to variations in cell proliferation. The resistant sub-cell line grew markedly slower than the parental cell line; the Td of HNE1/CDDP cells was lower than that of HNE1 cells, which may be caused by the existence of non-cycling 
A

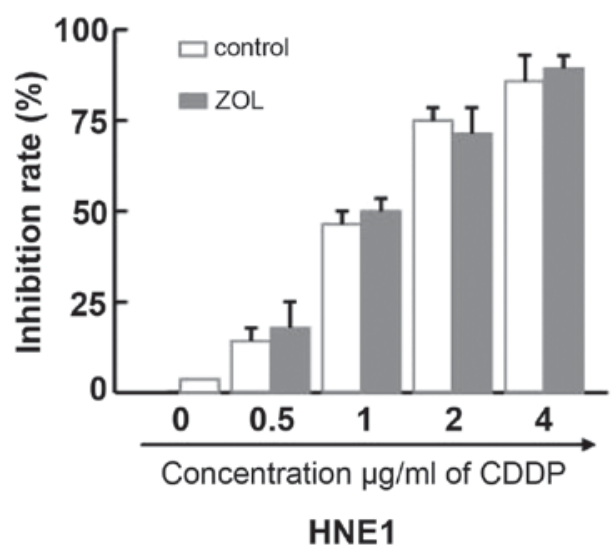

B

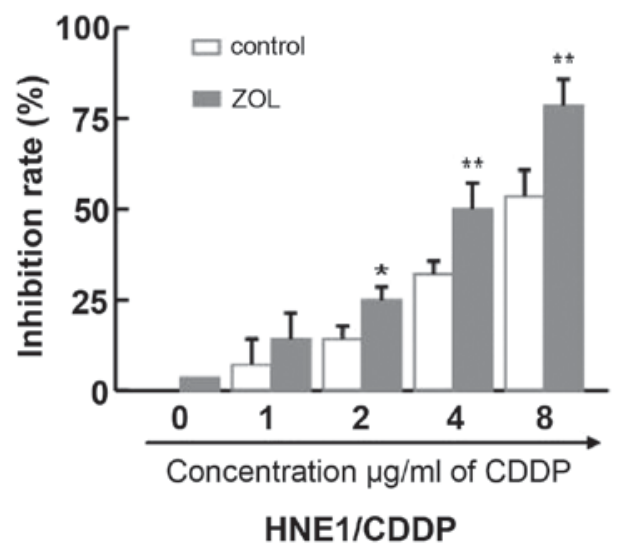

Figure 3. Effect of ZOL on the CDDP-sensitivity of nasopharyngeal carcinoma cells. (A) HNE1 and (B) HNE1/CDDP cells were pretreated with vehicle control (PBS) or ZOL $(2.90 \mu \mathrm{g} / \mathrm{ml})$ for $6 \mathrm{~h}$ prior to exposure to CDDP at indicated doses. After $48 \mathrm{~h}$, the drug sensitivity was measured using MTT assays. Data are expressed as the mean \pm standard deviation of three independent experiments. ${ }^{* *} \mathrm{P}<0.05,{ }^{* *} \mathrm{P}<0.01$ vs. the control group. ZOL, zoledronic acid; CDDP, cisplatin.
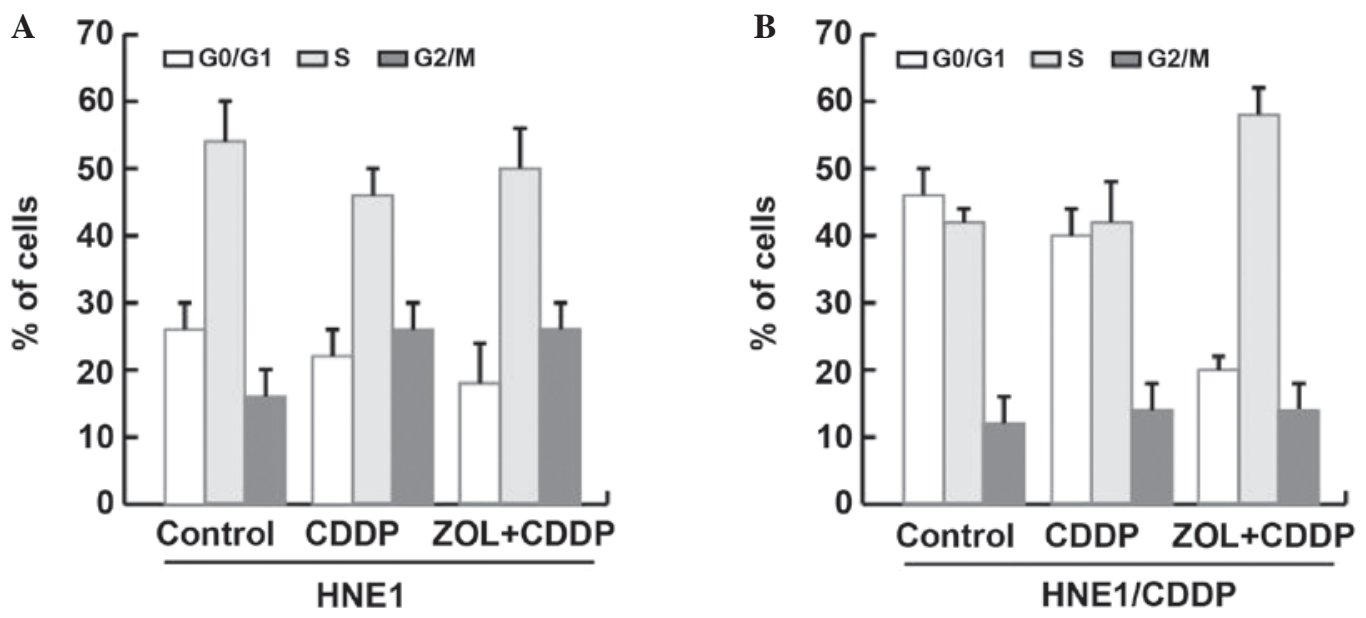

Figure 4. Effects of ZOL on the cell cycle distribution of nasopharyngeal carcinoma cells. (A) HNE1 and (B) HNE1/CDDP cells were incubated with ZOL $(2.90 \mu \mathrm{g} / \mathrm{ml})$ for $6 \mathrm{~h}$ prior to exposure to CDDP $(1 \mu \mathrm{g} / \mathrm{ml})$, after which they were harvested, stained with propidium iodide and analyzed by flow cytometry. Data are shown as the mean \pm standard deviation of three independent experiments. ZOL, zoledronic acid; CDDP, cisplatin.

dormant cells (22-24). The balance between cell cycle arrest, which promotes DNA repair and survival, and cell death following chemotherapy is critical $(23,24)$. Therefore, the present study further investigated whether dysregulation of the cell cycle could lead to drug resistance, thereby reducing the efficacy of chemotherapy. As expected, a prominent G0/G1 phase arrest was observed in CDDP-resistant cells, as compared with the parental cells, when they were exposed to CDDP. This result was consistent with previous reports that cancer cells were resistant to chemotherapy when in the G0/G1 phase, at which time they have an enhanced capacity for DNA damage repair $(23,24)$. Further elucidation of the effects of chemotherapy on the cell cycle may allow this cell cycle-mediated resistance to be overcome.

Significant efforts have been made to reverse the development of resistance in response to cancer chemotherapy. In previous studies, molecules or drugs targeting P-glycoprotein were used to inhibit the drug transporter in order to resensitize cancer cells, and the concept of combining chemotherapeutic agents to increase cytotoxic efficacy has evolved over the last few decades $(11,14,22,23)$. Among the chemotherapeutic agents, ZOL has exhibited promising sensitizing activity to be used in combination with chemotherapy and radiotherapy for cancer treatment (17-19). Notably, ZOL exhibited excellent activities against drug-resistant cancer cells $(25,26)$. The present study investigated the effects of a low concentration of ZOL on CDDP-resistant NPC cells; the low dose was selected as the peak plasma levels of ZOL was 1-2 $\mu \mathrm{M}$ (17). In addition, this low dose of ZOL demonstrated chemosensitizing effects while decreasing the side effects and complications. Notably, pretreatment with ZOL significantly reversed the CDDP resistance of NPC cells. This chemosensitization effect may have been due to $\mathrm{ZOL}$ inducing $\mathrm{S}$ phase cell cycle arrest, following a decrease in the percentage of cells in the G0/G1 phase and subsequent promotion of apoptosis, consistent with previous reports $(17-19,25,27)$. The lower percentage of CDDP-resistant HNE1 cells in the G0/G1 phase may indicate a reduced capacity for DNA damage repair, which is considered a mechanism to overcome drug resistance. 
CDDP
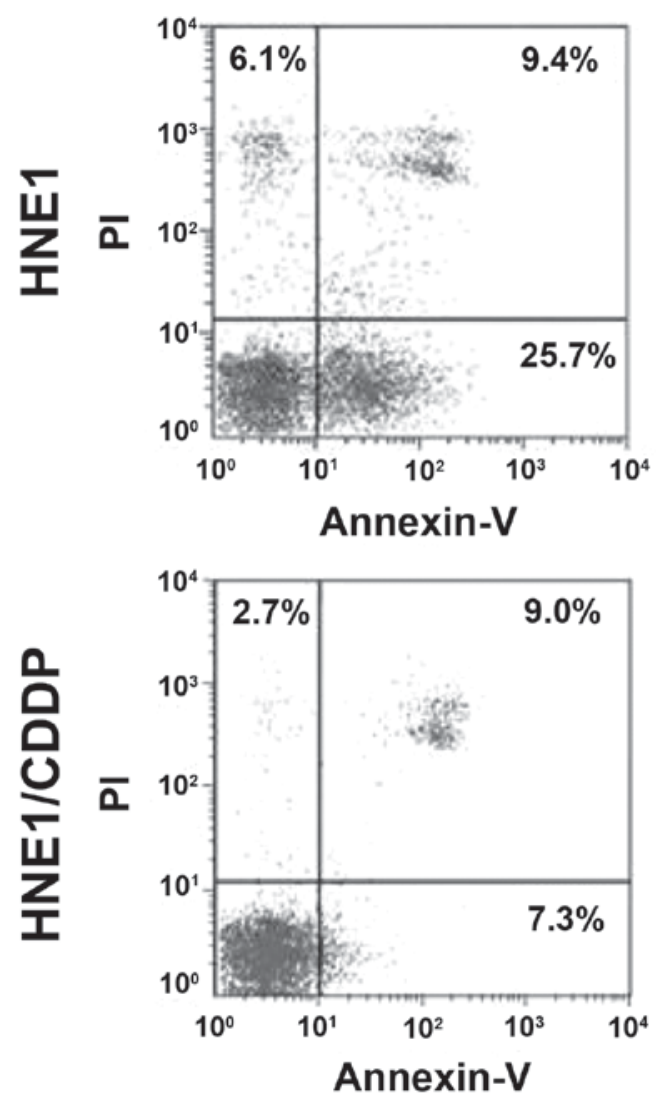

CDDP+ZOL
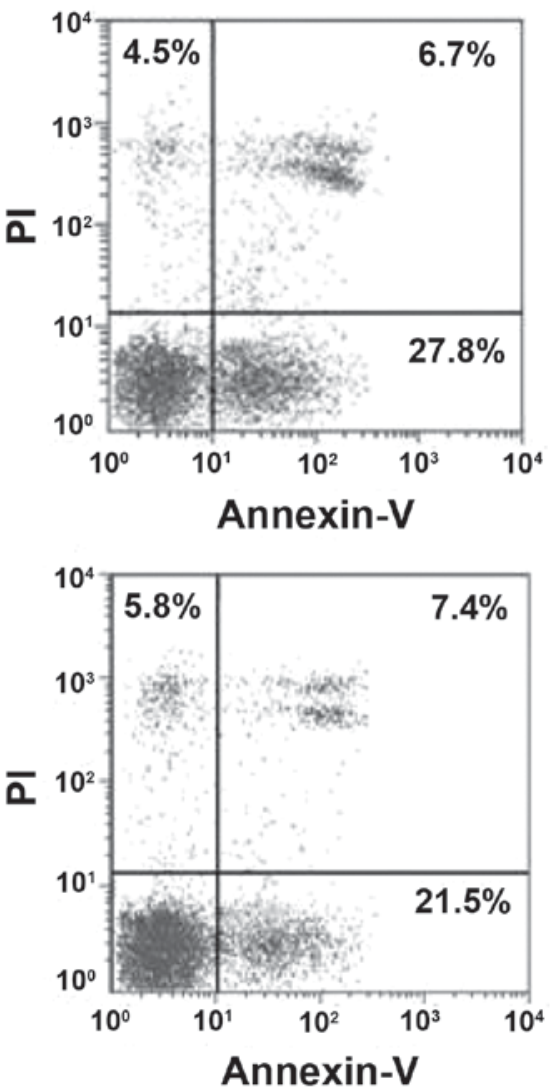

Figure 5. ZOL modulates CDDP-induced cell apoptosis. HNE1 and HNE1/CDDP cells were pretreated with ZOL ( $2.90 \mu \mathrm{g} / \mathrm{ml})$ for $6 \mathrm{~h}$ and exposed to $1 \mu \mathrm{g} / \mathrm{ml}$ CDDP for an additional $24 \mathrm{~h}$. Cells were then subjected to Annexin V/PI staining and analyzed by flow cytometry. ZOL, zoledronic acid; CDDP, cisplatin; PI, propidium iodide.

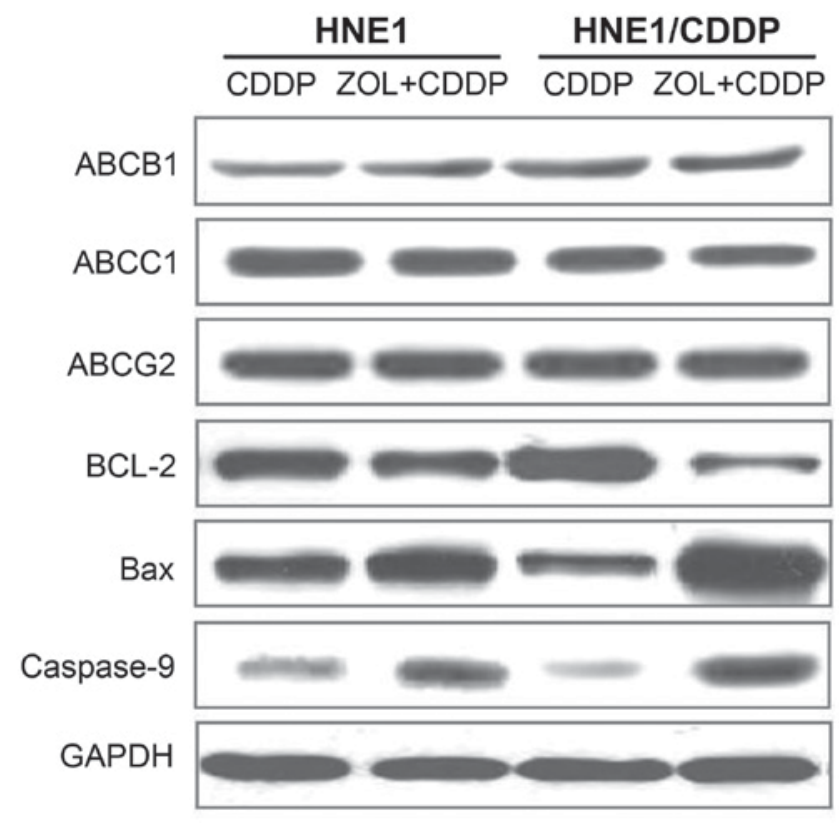

Figure 6. Western blot analysis of drug resistance and apoptosis-related genes. HNE1 and HNE1/CDDP cells were pretreated with ZOL $(2.90 \mu \mathrm{g} / \mathrm{ml})$ for $6 \mathrm{~h}$ prior to exposure to $1 \mu \mathrm{g} / \mathrm{ml} \mathrm{CDDP}$ for $24 \mathrm{~h}$, after which the protein expression levels of various genes were assessed by western blotting. ZOL, zoledronic acid; CDDP, cisplatin; ABCB1, ATP-binding cassette subfamily B member 1; ABCC1, ATP-binding cassette subfamily C member 1; ABCG2, ATP-binding cassette subfamily G member 2; BCL-2, B-cell lymphoma-2; BAX, BCL-2-associated $X$ protein.
BAX and caspase-9 expression was upregulated and BCL-2 expression was downregulated in CDDP-resistant cells pretreated with ZOL, suggesting that activation of the mitochondrial apoptotic pathway may be a key molecular mechanism underlying the chemosensitization activity of ZOL. Mitochondrial apoptosis is usually dependent on the release of cytochrome $c$, the activation of the caspase family and involvement of the Bcl-2 family (28). This finding also suggested that ZOL in combination with CDDP could exert a synergistic inhibitory effect on multidrug-resistant NPC cells. Notably, this synergistic effect was only observed in CDDP-resistant NPC cells, but not in the parental cells. To the best of our knowledge, this is the first study to report that ZOL reverses the CDDP-resistant phenotype in cancer cells, and that pretreatment with ZOL prior to CDDP may be a potential therapeutic strategy to overcome chemoresistance in NPC.

Another well-documented mechanism underlying CDDP resistance in cancer cells is the increased efflux of CDDP by the $\mathrm{ABC}$ transporter protein family, including MDR1/ABCB1 (P-glycoprotein), MRP1/ABCC1 and BCRP/ABCG2 (20,29). However, we failed to detect the dysregulated expression of these genes in CDDP-resistant cells following pretreatment with ZOL.

In conclusion, the present study established a CDDP-resistant NPC cell line and further illustrated the potential mechanism underlying CDDP resistance. In addition, the 
results of the present study suggested that the use of a combination of ZOL and CDDP may overcome the rate of drug resistance and further improve the clinical efficacy of CDDP with an acceptable clinical toxicity, which may have a significant impact on the therapeutic approach to NPC.

\section{Acknowledgements}

The authors of the present study would like to thank Mr. Zhen Zhang at the Department of Biochemistry and Molecular Biology of the University of Kansas Medical Center (Lawrence, KS, USA) for his careful reading of the manuscript and suggestions. This study was supported by the Science and Technology Planning Project of Henan Province, China (grant no. 142102310464), the Key Research Foundation of Higher Education of Henan Province, China (grant no. 15B320003), the Annual Natural Science Foundation of Luohe Medical College, the Natural Science Foundation of Hubei Province (grant no. 2014CFC1154), the Foundation of Medical College of Hubei University of Arts and Science (grant no. YXKY 201402) and the Scientific Research Foundation for Doctoral Program of Hubei University of Arts and Science.

\section{References}

1. Ho JH: An epidemiologic and clinical study of nasopharyngeal carcinoma. Int J Radiat Oncol Biol Phys 4: 182-198, 1978.

2. Wei WI and Sham JS: Nasopharyngeal carcinoma. Lancet 365: 2041-2054, 2005.

3. McDermott AL, Dutt SN and Watkinson JC: The aetiology of nasopharyngeal carcinoma. Clin Otolaryngol Allied Sci 26: 82-92, 2001.

4. Feng Z, Xu S, Liu M, Zeng YX and Kang T: Chk1 inhibitor Gö6976 enhances the sensitivity of nasopharyngeal carcinoma cells to radiotherapy and chemotherapy in vitro and in vivo. Cancer Lett 297: 190-197, 2010.

5. Al-Sarraf M, LeBlanc M, Giri PG, Fu KK, Cooper J, Vuong T, Forastiere AA, Adams G, Sakr WA, Schuller DE and Ensley JF: Chemoradiotherapy versus radiotherapy in patients with advanced nasopharyngeal cancer: Phase III randomized Intergroup study 0099. J Clin Oncol 16: 1310-1317, 1998.

6. Portugal J, Bataller M and Mansilla S: Cell death pathways in response to antitumor therapy. Tumori 95: 409-421, 2009.

7. Wang X, Masters JR, Wong YC, Lo AK and Tsao SW: Mechanism of differential sensitivity to cisplatin in nasopharyngeal carcinoma cells. Anticancer Res 21: 403-408, 2001.

8. Cheung HW, Jin DY, Ling MT, Wong YC, Wang Q, Tsao SW and Wang X: Mitotic arrest deficient 2 expression induces chemosensitization to a DNA-damaging agent, cisplatin, in nasopharyngeal carcinoma cells. Cancer Res 65: 1450-1458, 2005.

9. Shannon KM: Resistance in the land of molecular cancer therapeutics. Cancer Cell 2: 99-102, 2002.

10. Liu T, Xu F, Du X, Lai D, Liu T, Zhao Y, Huang Q, Jiang L, Huang W, Cheng W and Liu Z: Establishment and characterization of multi-drug resistant, prostate carcinoma-initiating stem-like cells from human prostate cancer cell lines 22RV1. Mol Cell Biochem 340: 265-273, 2010.

11. Wang J, Wang H, Zhao L, Fan S, Yang Z, Gao F, Chen L, Xiao GG, Molnár J and Wang Q: Down-regulation of P-glycoprotein is associated with resistance to cisplatin and VP-16 in human lung cancer cell lines. Anticancer Res 30: 3593-3598, 2010.
12. Peng X, Li W and Tan G: Reversal of taxol resistance by cisplatin in nasopharyngeal carcinoma by upregulating thromspondin-1 expression. Anticancer Drugs 21: 381-388, 2010.

13. Low SY, Tan BS, Choo HL, Tiong KH, Khoo AS and Leong CO: Suppression of BCL-2 synergizes cisplatin sensitivity in nasopharyngeal carcinoma cells. Cancer Lett 314: 166-175, 2012.

14. Pan Y,Zhou F,Zhang R and Claret FX: Stat 3 inhibitor Stattic exhibits potent antitumor activity and induces chemo- and radio-sensitivity in nasopharyngeal carcinoma. PLoS One 8: e54565, 2013.

15. Catanzaro D, Vianello C, Ragazzi E, Caparrotta L and Montopoli M: Cell cycle control by natural phenols in cisplatin-resistant cell lines. Nat Prod Commun 9: 1465-1468, 2014.

16. Xie SM, Fang WY, Liu TF, Yao KT and Zhong XY: Association of $A B C C 2$ and CDDP-resistance in two sublines resistant to CDDP derived from a human nasopharyngeal carcinoma cell line. J Oncol 2010: 915046, 2010.

17. You Y, Liu J, Wang Z, Zhang Y, Ran Y, Guo X, Liu H and Wang $\mathrm{H}$ : The enhancement of radio-sensitivity in human esophageal squamous cell carcinoma cells by zoledronic acid and its potential mechanism. Cytotechnology 66: 17-25, 2014.

18. Koto K, Murata H, Kimura S, Horie N, Matsui T, Nishigaki Y, Ryu K, Sakabe T, Itoi M, Ashihara E, et al: Zoledronic acid inhibits proliferation of human fibrosarcoma cells with induction of apoptosis, and shows combined effects with other anticancer agents. Oncol Rep 24: 233-239, 2010.

19. Zhao M, Tominaga Y, Ohuchida K, Mizumoto K, Cui L, Kozono S, Fujita H, Maeyama R, Toma $\mathrm{H}$ and Tanaka M: Significance of combination therapy of zoledronic acid and gemcitabine on pancreatic cancer. Cancer Sci 103: 58-66, 2012.

20. Yan LH, Wang XT, Yang J, Kong FB, Lian C, Wei WY, Luo W, Xie YB and Xiao Q: Reversal of multidrug resistance in gastric cancer cells by E2F-1 downregulation in vitro and in vivo. J Cell Biochem 115: 34-41, 2014.

21. Liu J, Yang L, Zhang J, Zhang J, Chen Y, Li K, Li Y, Li Y, Yao L and Guo G: Knock-down of NDRG2 sensitizes cervical cancer Hela cells to cisplatin through suppressing Bcl-2 expression. BMC Cancer 12: 370, 2012

22. Siddik ZH: Cisplatin: Mode of cytotoxic action and molecular basis of resistance. Oncogene 22: 7265-7279, 2003.

23. Blagosklonny MV: Targeting cancer cells by exploiting their resistance. Trends Mol Med 9: 307-312, 2003.

24. Shah MA and Schwartz GK: Cell cycle-mediated drug resistance: An emerging concept in cancer therapy. Clin Cancer Res 7: 2168-2181, 2001

25. Moriceau G, Ory B, Mitrofan L, Riganti C, Blanchard F, Brion R, Charrier C, Battaglia S, Pilet P, Denis MG, et al: Zoledronic acid potentiates mTOR inhibition and abolishes the resistance of osteosarcoma cells to RAD001 (Everolimus): Pivotal role of the prenylation process. Cancer Res 70: 10329-10339, 2010.

26. Koizumi M, Nakaseko C, Ohwada C, Takeuchi M, Ozawa S, Shimizu N, Cho R, Nishimura M and Saito Y: Zoledronate has an antitumor effect and induces actin rearrangement in dexamethasone-resistant myeloma cells. Eur J Haematol 79: 382-391, 2007.

27. Li XY, Lin YC, Huang WL, Lin W, Wang HB, Lin WZ and Lin SL: Zoledronic acid inhibits human nasopharyngeal carcinoma cell proliferation by activating mitochondrial apoptotic pathway. Med Oncol 29: 3374-3380, 2012.

28. Okuno S, Shimizu S, Ito T, Nomura M, Hamada E, Tsujimoto Y and Matsuda $\mathrm{H}$ : Bcl-2 prevents caspase-independent cell death. J Biol Chem 273: 34272-34277, 1998.

29. Han T, Zhu X, Wang J, Zhao H, Ma Q, Zhao J, Qiu X and Fan Q: Establishment and characterization of a cisplatin-resistant human osteosarcoma cell line. Oncol Rep 32: 1133-1139, 2014. 